\title{
Vitamin D stimulates multiple microRNAs to inhibit CRH and other pro-labor genes in human placenta
}

\author{
Bingbing Wang', Mayra Cruz Ithier', Nataliya Parobchak', Stacy M Yadava', Jay Schulkin² and Todd Rosen' \\ ${ }^{1}$ Division of Maternal-Fetal Medicine, Department of Obstetrics, Gynecology and Reproductive Sciences, Rutgers Robert Wood Johnson Medical School, \\ New Brunswick, New Jersey, USA \\ ${ }^{2}$ Department of Obstetrics, Gynecology, University of Washington, Seattle, Washington, USA \\ Correspondence should be addressed to B Wang or T Rosen: wangbi@rwjms.rutgers.edu or rosentj@rwjms.rutgers.edu
}

\begin{abstract}
Maternal vitamin D deficiency is linked to adverse pregnancy outcomes including spontaneous preterm birth (SPB). Placental corticotropin-releasing hormone (CRH) has been proposed to be part of a clock that governs the length of gestation in humans, with elevated maternal serum levels predicting early delivery. In this study, we test the hypothesis that vitamin D could contribute to the prevention of preterm labor by inhibiting $\mathrm{CRH}$ and other pro-labor mediators. The biological activity of vitamin $\mathrm{D}$ occurs via two pathways: non-genomic and genomic responses, both of which involve binding of 1,25-dihydroxyvitamin $D(1,25(\mathrm{OH}) 2 \mathrm{D})$, the active metabolite of vitamin $D$ binding to the vitamin $D$ receptor (VDR). By using chromatin immunoprecipitation followed by sequencing (ChIP-seq), we found that 1,25(OH)2D stimulates association of VDR with a number of miRNA genes including MIR181B2 and MIR26B, and their mature products miR-181b-5p and miR-26b-5p are predicted to target $C R H$ and cyclooxygenase-2

(COX-2) mRNA at 3'-untranslated region (UTR), respectively. We performed RT-qPCR analysis to validate that expression of mature miR-181b-5p and miR-26b-5p in term human syncytiotrophoblast increased in response to treatment with 1,25(OH)2D. miR-181b-5p- or miR-26b-5p-mediated inhibition of CRH or COX-2 was further assessed by the use of miRNA mimics/inhibitors and a luciferase reporter assay. Taken together, this study has identified novel mechanisms by which vitamin D downregulates pro-labor genes and could lower the risk of preterm delivery.
\end{abstract}

\author{
Key Words \\ - vitamin D \\ - microRna \\ - $\mathrm{CRH}$ \\ - pro-labor genes \\ - placenta
}

\section{Introduction}

Vitamin D deficiency has been linked to an increased risk of preterm delivery. A cohort study by Bodnar and colleagues using specimens obtained from the US Collaborative Perinatal Project found a link between vitamin $\mathrm{D}$ deficiency and preterm infants in nonwhite mothers. Placental histology showed that some of these preterm births were inflammation-mediated while other cases occurred in the absence of detectable placental pathology (1). The same group studied a separate case-cohort study on an entirely different group of pregnant women with banked serum samples and found that sufficient vitamin D levels protected against spontaneous preterm birth (2). There is moderate evidence that from three randomized controlled trials involving 477 women that vitamin D supplementation reduces preterm birth risk (3). Maternal vitamin D deficiency and elevated CRH have also been linked to a number of adverse pregnancy outcomes including preeclampsia, fetal growth restriction and gestational diabetes mellitus (4).

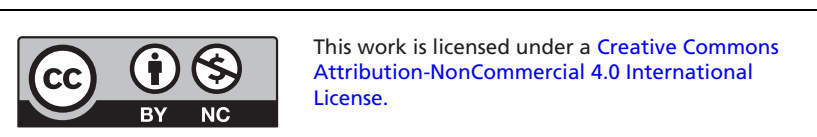


During pregnancy, the human placenta produces a large amount of corticotropin-releasing hormone (CRH) (5). Maternal plasma CRH levels increase exponentially as pregnancy advances, peaking at the time of delivery. Women who deliver preterm have increased levels of CRH early in pregnancy, while those who deliver post-term tend to have lower CRH levels (6). The link between increased CRH levels and preterm delivery has been demonstrated by a number of investigators $(7,8,9)$. Because of this, $\mathrm{CRH}$ has been suggested to be involved in the regulation of a placental clock, acting in concert with other factors to regulate a cascade of physiological events leading to parturition (6).

A hypothesis that has been proposed is that vitamin D might inhibit $C R H$ gene expression in placenta tissue and thereby decrease the vulnerability for prematurity in childbirth $(10,11)$. Indeed, Mohamed and colleagues have suggested that vitamin $\mathrm{D}$ and $\mathrm{CRH}$ might be linked to preterm labor and birth, because they found a correlation between low 25(OH)D and elevated CRH levels in women who delivered preterm (10).

One of the critical events that occurs in the initiation of parturition is the induction of prostaglandin synthesis in both fetal and maternal tissues. Prostaglandins play a role in the onset of effective uterine contractions, cervical ripening and increasing uterine receptivity to oxytocin (12). It has been previously shown that both $C R H$ and $C O X-2$, a key gene responsible for prostaglandin synthesis, are regulated similarly in the human placenta (13). As such, we chose to explore the potential mechanisms of vitamin $\mathrm{D}$ action on both $\mathrm{CRH}$ and $\mathrm{COX}-2$.

The biological activity of vitamin D occurs via two pathways: non-genomic and genomic responses, both of which involve binding of 1,25-dihydroxyvitamin D $(1,25(\mathrm{OH}) 2 \mathrm{D})$, the active metabolite of vitamin $\mathrm{D}$ and the vitamin D receptor (VDR), a member of the superfamily of nuclear receptor for steroid hormones (14). Prevention of bacterial infection-mediated SPB by vitamin $\mathrm{D}$ is likely dependent on its non-genomic response, because 1,25(OH)2D-simulated VDR can physically interact with IKB kinase $\beta$ (IKK $\beta$ ) to block lipopolysaccharide (LPS)induced NF-KB activation, which induces production of a variety of proinflammatory cytokines for initiation of labor $(15,16)$. In contrast, vitamin $D$ supplementation prevents SPB cases not related to inflammation most likely via the genomic mechanism, because a recent study in the myometrium revealed that vitamin $\mathrm{D}$ can inhibit multiple labor-associated genes including connexin 43 and prostaglandin receptors (17), which are not NF-KB target genes.
In the current study, we tested the hypothesis that vitamin D may inhibit pro-labor genes via a genomic response. We worked with primary cultures of human syncytiotrophoblast (STB), the source of maternal serum $\mathrm{CRH}$ (6) and one of major sites for COX-2 production during human pregnancy (18). Our results reveal a novel mechanism underlying vitamin $\mathrm{D}$ regulation of CRH and COX-2 genes in the placenta and contribute to our understanding of the potential link between vitamin $\mathrm{D}$ deficiency and preterm delivery. We also lay the groundwork for future studies of how vitamin D may regulate other genes potentially involved in the initiation of human labor and preterm labor.

\section{Materials and methods}

\section{Placental specimens}

We collected the placenta from healthy women with estimated gestational age of 38 and 40 weeks who were delivered by elective Cesarean section (C-section). Women with complications of pregnancies, including diabetes, hypertension, autoimmune diseases, infection, fetal growth restriction and preeclampsia, were excluded from the study.

This study was approved by the Institutional Review Board of Rutgers University (\#Pro20150001445). Because specimens used for the study would normally be discarded, there was no risk to the patient or her pregnancy from study procedures, and it was not deemed appropriate to approach patients on Labor and Delivery for consent because potential subjects might be under duress, the IRB granted a waiver of consent for this study.

\section{Purification of cytotrophoblast}

Briefly, villous tissue fragments from the entire placenta were subject to enzymatic digestions in a solution containing $0.25 \%$ trypsin, $0.2 \%$ deoxyribonuclease I, 25 mM HEPES, $2 \mathrm{mM} \mathrm{CaCl}_{2}$ and $0.8 \mathrm{mM} \mathrm{MgSO}_{4}$ in $1 \times$ Hanks' $^{\prime}$ balanced salt solution at $37^{\circ} \mathrm{C}$ followed by filtration of $100-\mu \mathrm{m}$ sieve. Cells were pelleted and resuspended in DMEM/F-12 with 10\% fetal bovine serum (FBS). We used a discontinuous density gradient of Percoll (50/45/35/30\%) by centrifuging at $1000 \boldsymbol{g}$ at room temperature for $20 \mathrm{~min}$. Target cells at the interface of fractions of 35/45\% were collected and further immunopurified by an approach of negative selection with use of human CD9 and CD45 antibodies (BD Biosciences, San Diego, CA, USA) and

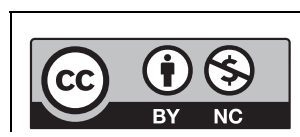

This work is licensed under a Creative Commons Attribution-NonCommercial 4.0 International License. 
Dynabeads (Invitrogen). Cells in the supernatant that were separated from Dynabeads with contaminated cells were pelleted, resuspended in DMEM/F-12 plus 10\% FBS, plated at a density of $2.5-3 \times 10^{6} / \mathrm{cm}^{2}$ and maintained at $37^{\circ} \mathrm{C}$ and $5 \% \mathrm{CO}_{2}$ at least for $48 \mathrm{~h}$ to spontaneously differentiate into STB prior to further analysis.

\section{ChIP-sequencing (ChIP-seq)}

A total of approximately $1 \times 10^{7}$ of STB cells were cross-linked with $1 \%$ formaldehyde for $5 \mathrm{~min}$ at room temperature, and the reaction was stopped by the addition of $1 \times$ glycine. Cells were lysed in ChIP lysis buffer (50 mM HEPES-KCl, pH 7.5; $140 \mathrm{mM} \mathrm{NaCl} ; 1 \mathrm{mM}$ EDTA; $1 \%$ Triton X-100; 0.1\% sodium deoxycholate; $0.1 \%$ SDS) with freshly added $1 \times$ protease inhibitor cocktail (Roche Applied Science) and then sonicated to shear chromatin into 150- to 200-bp fragments. Chromatin was then immunoprecipitated with individual ChIP-grade anti-VDR antibody (ThermoFisher Scientific) at $5 \mu \mathrm{g} / 25 \mu \mathrm{g}$ chromatin and incubated with protein $G$ agarose beads (Roche Applied Science). Immunoprecipitates were treated with Proteinase K and DNA fragments were recovered by phenol/chloroform extraction and ethanol precipitation.

Concentrations of DNA were determined by Qubit Fluorometric (Invitrogen) and at least $10 \mathrm{ng} /$ per sample were submitted for ChIP-seq with Illumina HiSeq platform and $1 \times 50 \mathrm{bp}$ configuration (GENEWIZ, NJ, USA). Approximately 12 million paired-end reads/per sample were requested.

\section{Gene silencing}

siRNA transfection was performed as previously detailed using transfection reagent Lipofectamine2000 (Invitrogen) with use of FlexiTube siRNAs target VDR or miRNA inhibitors as indicated (Qiagen) $(19,20)$. Total RNAs were isolated from cells and analyzed by RT-qPCR. Each experiment was repeated in three individual specimens.

\section{Reverse transcription quantitative PCR (RT-qPCR)}

We extracted total RNAs by means of TRIzol (Invitrogen). For assessment of mRNA levels, cDNA synthesis was prepared by the oligo-dT primer method using the Superscript II Reverse Transcription kit (Thermo Fischer Scientific). PCR was performed using a StepOne Plus Real Time PCR System (Applied Biosystems) and power SYBR green PCR master (ThermoFisher Scientific). PCR primers (c) 2018 The authors Published by Bioscientifica Ltd (forward/reverse) included CRH, 5'-GCAGTTAGCACAGCA AGCTCAC-3'/5'-CAAATGGCATAAGAGCAGCG-3'; COX-2, 5 ' - T GA GCATCTACG GTT T G CT G - 3' / 5 ' - T GCTTG TCTGGAACAACTGC-3' and GAPDH, 5'-CTCCCGCTT CGCTCTCTG-3'/5'-CTGGCGACGCAAAAGAAG-3'.

We used the miScript PCR System (Qiagen) for quantification of mature miRNAs with a universal primer and miRNA-specific primer included in the kit according to the manufacture's protocol. U6 snRNA (5'-CTCGCTTCGGCAGCACA-3' / 5' -AACGCTTCAC GAATTTGCGT-3') served as the internal control.

\section{Western blot}

The protein samples were separated on SDS-10\% PAGE and transferred onto PVDF membranes (Bio-Rad). Membranes were blocked in 5\% nonfat milk powder in PBST (10 mM phosphate buffer, pH 7.2; $150 \mathrm{mM} \mathrm{NaCl}$; and $0.1 \%$ Tween-20) for $60 \mathrm{~min}$, washed twice with PBST, and incubated with antibodies as indicated in $1 \%$ nonfat milk powder-PBST at $4^{\circ} \mathrm{C}$ overnight. Membranes were washed three times with PBST, incubated with horseradish peroxidase-conjugated secondary antibodies at 1:5000 in 1\% nonfat milk powder-PBST and developed by Immun-Star HRP Substrate (Bio-Rad). The blots were visualized either by autoradiography or scanned by Chemiluminescence Western Blot Scanner (C-DiGit, LI-COR, NE, USA).

\section{Dual-luciferase assay}

All firefly luciferase (FL) reporter constructs were made available by GeneScript (NJ, USA) based on PCD FL6X plasmid (\#12566, Addgene, MA, USA). The individual FL reporter construct was co-tranfected with a Renilla luciferase reporter construct, pRL-CMV (Promega) with use of Lipofectamine 2000 (Invitrogen) as previously described (19). Dual-luciferase assay was read on Infinite M200 Pro microplate reader (TECAN, USA) according to the manufacturer's protocol (Promega).

\section{Statistical analysis}

Each experiment was repeated a minimum of three times. Data (bars) are presented as mean \pm standard deviation (s.D.). One-way ANOVA was used to compare among $\geq 3$ groups. Student $t$ test was used to compare the control with each individual test group. $P<0.05$ was considered statistically significant. https://ec.bioscientifica.com

https://doi.org/10.1530/EC-18-0345

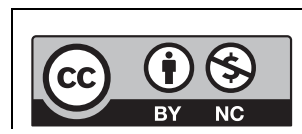

This work is licensed under a Creative Commons Attribution-NonCommercial 4.0 International License. 
Table 1 Summary of genomic loci associated with VDR stimulated by $1,25(\mathrm{OH}) 2 \mathrm{D}(P<0.05)$.

\begin{tabular}{|c|c|c|}
\hline & $\begin{array}{l}\text { Number of } \\
\text { genomic loci }\end{array}$ & Chromosomes \\
\hline Non-coding RNA genes & 1112 & $1-22, X, Y$ \\
\hline MicroRNAs & 542 & $1-22, X, Y$ \\
\hline Others & 570 & $1-22, X, Y$ \\
\hline Protein-coding genes & 148 & $1-22$ \\
\hline Pseudogenes & 326 & $1-22, X, Y$ \\
\hline Total & 1586 & \\
\hline
\end{tabular}

\section{Results}

Genomic sites targeted by 1,25(OH)2D-simulated VDR in human STB

We first used chromatin immunoprecipitation sequencing (ChIP-seq) to perform a genome-wide profile of DNAbinding sites targeted by 1,25(OH)2D-simulated VDR in human STB. Primary cytotrophoblast were purified from the placenta from normal healthy individuals and cultured for at least $48 \mathrm{~h}$ to spontaneously differentiate into STB, which in turn were exposed to $1,25(\mathrm{OH}) 2 \mathrm{D}$ at $10 \mu \mathrm{M}$ for additional $24 \mathrm{~h}$. DMSO was used as the vehicle control. Cells were cross-linked with $1 \%$ formaldehyde and then sonicated to shear chromatin into 100-200bp DNA fragments, and ChIP was conducted with use of ChIP-grade VDR antibody. Normal rabbit IgG was used as the input control. Recovered DNAs were used for preparation of DNA libraries and in turn sequenced on an Illumina platform.

Accumulated evidence has implicated a key regulatory function for miRNAs in gene expression. In mammals, miRNAs regulate about $30 \%$ of all protein-coding genes, typically in a negative manner, and have been shown to participate in the regulation of almost every cellular process examined so far (21). As shown in Table 1, there were a total of 1586 genomic sites targeted by $1,25(\mathrm{OH}) 2 \mathrm{D}$ simulated VDR in human STB, which include 1112 sites for non-coding RNA genes and 148 for coding genes.
Remarkably, 1,25(OH)2D-simulated VDR was able to bind to a total of 542 miRNA genes (Supplementary Table 1, see section on supplementary data given at the end of this article), strongly suggesting a role of vitamin D-mediated regulation of target miRNA genes.

These data support that $1,25(\mathrm{OH}) 2 \mathrm{D}-\mathrm{VDR}$ stimulates transcription of miRNA genes by directly associating with their precursor DNA sequences as previously suggested (22).

Pro-labor genes are negatively regulated by miRNAs that are stimulated by 1,25(OH)2D-VDR

Next, we combined TargetScan (23), a web server that predicts biological targets of miRNAs by searching for the presence of sites that match the seed region of each miRNA, with miRBase (24), a biological database that acts as an archive of microRNA sequences and annotations, to annotate target genes by miRNAs.

In order to identify miRNAs that target $C R H$ or COX-2 gene, we first input CRH or PTGS2, an official gene symbol for COX-2, as the gene to be studied in TargetScan. There were a total of 130 miRNAs putatively targeting $\mathrm{CRH}$, however, only 15 of which were stimulated by $1,25(\mathrm{OH}) 2 \mathrm{D}-\mathrm{VDR}$ in human STB (Supplementary Tables 1 and 2). We further assessed these 15 miRNAs against miRBase for confidence of annotation and found that miR-181b-5p was only the one of these 15 miRNAs with a defined annotation confidence validating it as a bona fide miRNA in humans. There were four miRNAs including miR-1297, miR-26b-5p, miR-26a-5p and miR-4465 that putatively target COX-2, and only miR-26b-5p was stimulated by $1,25(\mathrm{OH}) 2 \mathrm{D}-\mathrm{VDR}$. A search of miR-26b-5p in miRBase determined it to be a validated human miRNA.

We used a similar approach to determine if any miRNAs might target other pro-labor genes. The results of this analysis may be found in Table 2.

Table 2 Select microRNA genes stimulated by VDR, their mature RNAs and putative target genes involved in initiation of human labor.

\begin{tabular}{l} 
Gene symbol \\
\hline MIRLET7A1 \\
MIRLET7I \\
MIR26B \\
MIR29A \\
MIR29C \\
MIR302C \\
MIR130A \\
MIR181B2 \\
MIR183
\end{tabular}

Mature miRNAs
let-7a-5p
let-7i-5p
miR-26b-5p
miR-29a-3p
miR-29c-3p
miR-302c-3p
miR-130-3p
miR-181-5p
miR-183-5p

https://ec.bioscientifica.com https://doi.org/10.1530/EC-18-0345 (c) 2018 The authors Published by Bioscientifica Ltd

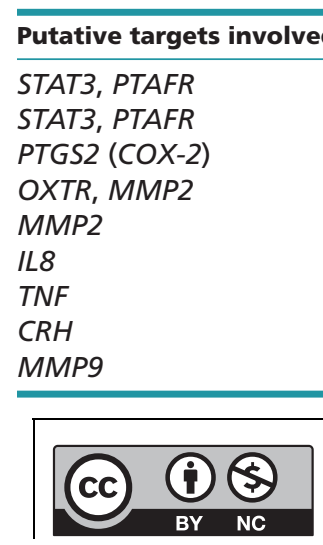

$\begin{array}{r}\hline \text { P value } \\ \hline 0.01 \\ <0.01 \\ <0.01 \\ <0.01 \\ 0.03 \\ <0.01 \\ <0.01 \\ <0.01 \\ <0.01 \\ \hline\end{array}$

This work is licensed under a Creative Commons Attribution-NonCommercial 4.0 International License. 


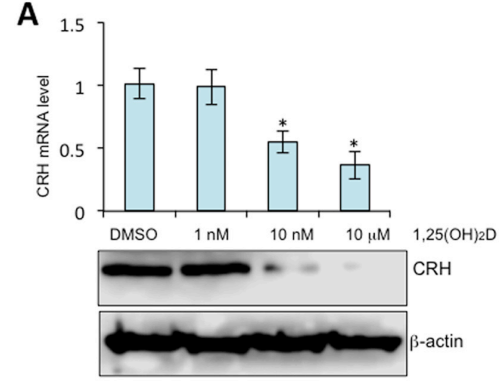

B

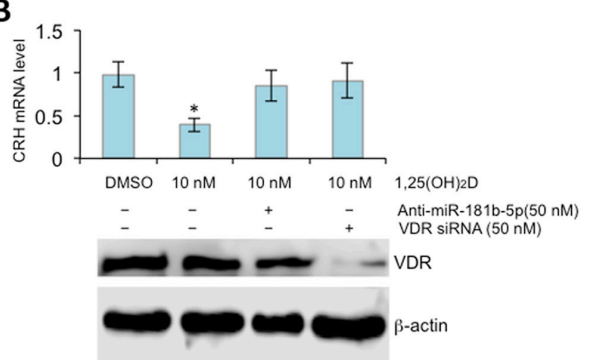

\section{Figure 1}

1,25(OH)2D stimulates miR-181b-5p to inhibit CRH in the human placenta. (A) Term STB were treated with 1,25(OH)2D at concentrations as indicated for $24 \mathrm{~h}$. mRNA and protein levels were assessed by RT-qPCR with normalization to GAPDH (top panel) or Western blot (bottom panel), respectively. Error bars were derived from three independent experiments. (B) Term STB were treated with $m i R-181 b-5 p$ anatagomirs (inhibitors) or VDR siRNA for $24 \mathrm{~h}$ followed by $1,25(\mathrm{OH}) 2 \mathrm{D}$ at final concentration of $10 \mathrm{nM}$ for additional $24 \mathrm{~h}$. mRNA and protein levels were assessed by RT-qPCR (top panel) or Western blot (bottom panel), respectively. Three independent experiments were performed.

\section{Vitamin D inhibits CRH via \\ VDR-miR-181b-5p pathway}

The primate placenta is unique in its ability to produce $\mathrm{CRH}$, which is considered to be part of placental clock that controls gestational length and parturition.

To assess whether vitamin D-upregulated miR-181b-5p can directly suppress CRH mRNA as predicted by the analysis depicted in Table 2, we first incubated 1,25(OH)2D with human STB and used RT-PCR to assess mRNA level changes. As shown in Fig. 1, 1,25(OH)2D exposure inhibited $\mathrm{CRH}$ in dose-dependent manner, and this repression was reversed when cells were treated with VDR siRNA or $m i R-181 b-5 p$ inhibitors. This data suggests that vitamin D downregulates $C R H$ by stimulating VDR, and in turn, expression of $m i R-181 b-5 p$.

\section{Vitamin D downregulates COX-2 via the VDR-miR-26b-5p pathway}

We incubated 1,25(OH)2D with human STB and used RT-PCR to assess mRNA level changes of $C O X-2$. As shown in Fig. 2, 1,25(OH)2D treatment led to a significant decrease

$\begin{array}{lr}\text { https://ec.bioscientifica.com } & \text { ○ } 2018 \text { The authors } \\ \text { https://doi.org/10.1530/EC-18-0345 } & \text { Published by Bioscientifica Ltd }\end{array}$

of COX-2, and such a repression was reversed when cells were treated with VDR siRNA or $m i R-26 b-5 p$ inhibitors. This data supports that vitamin $\mathrm{D}$ downregulates COX -2 by stimulating VDR, and in turn, expression of miR-26b-5p.

We further employed RT-qPCR to validate VDRstimulated expression of mature miR-181b-5p and $m i R-26 b-5 p$ by RT-qPCR with $m i R-21-5 p$ as the negative control (Fig. 3). Collectively, these data suggest that the protective effect of vitamin $\mathrm{D}$ against spontaneous preterm delivery is a consequence of genomic response at the $C R H$ and $C O X-2$ gene.

\section{miR-181b-5p and miR-26b-5p directly target CRH and COX-2, respectively}

We further assessed the ability of $m i R-181 b-5 p$ to bind to the 3 '-UTR of CRH or miR-26b-5p to bind to the 3 '-UTR of COX-2. To achieve this goal, we performed a luciferase reporter assay and observed a significant decrease in luciferase activity in the presence of $m i R-181 b-5 p$ (Fig. $4 \mathrm{~A}$ and B) or miR-26b-5p mimics in term STB compared with the controls
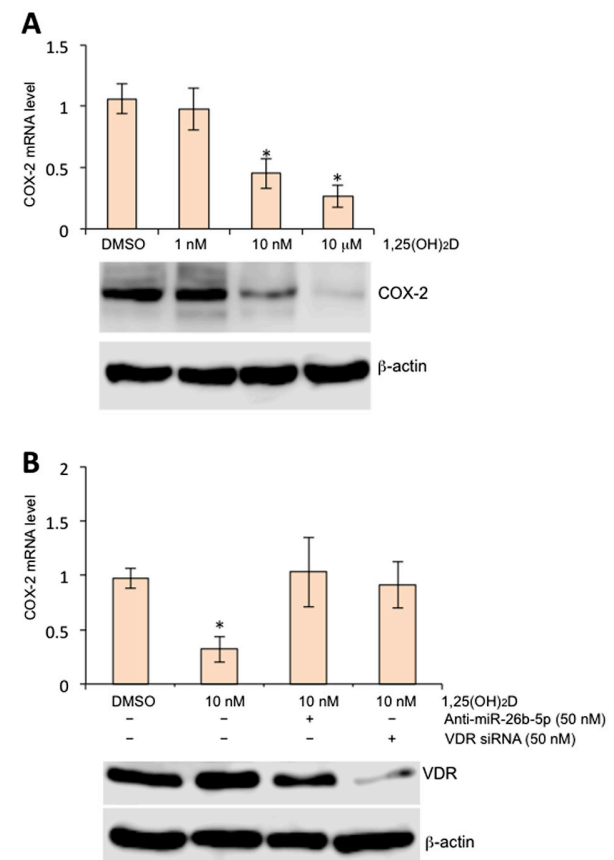

Figure 2

1,25(OH)2D stimulates miR-26b-5p to inhibit COX-2 in the human placenta. (A) Term STB were treated with 1,25(OH)2D at concentrations as indicated for $24 \mathrm{~h}$. mRNA and protein levels were assessed by RT-qPCR (top panel) or Western blot (bottom panel), respectively. Error bars were derived from three independent experiments. (B) Term STB were treated with miR-26b antagomirs or VDR siRNA for $24 \mathrm{~h}$ followed by $1,25(\mathrm{OH}) 2 \mathrm{D}$ at final concentration of $10 \mathrm{nM}$ for additional $24 \mathrm{~h}$. mRNA levels were assessed by RT-qPCR. Three independent experiments were performed.

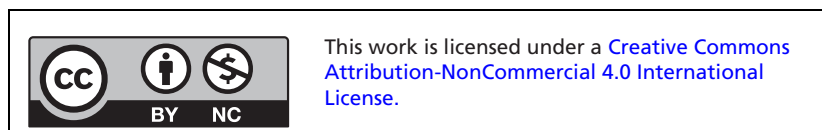




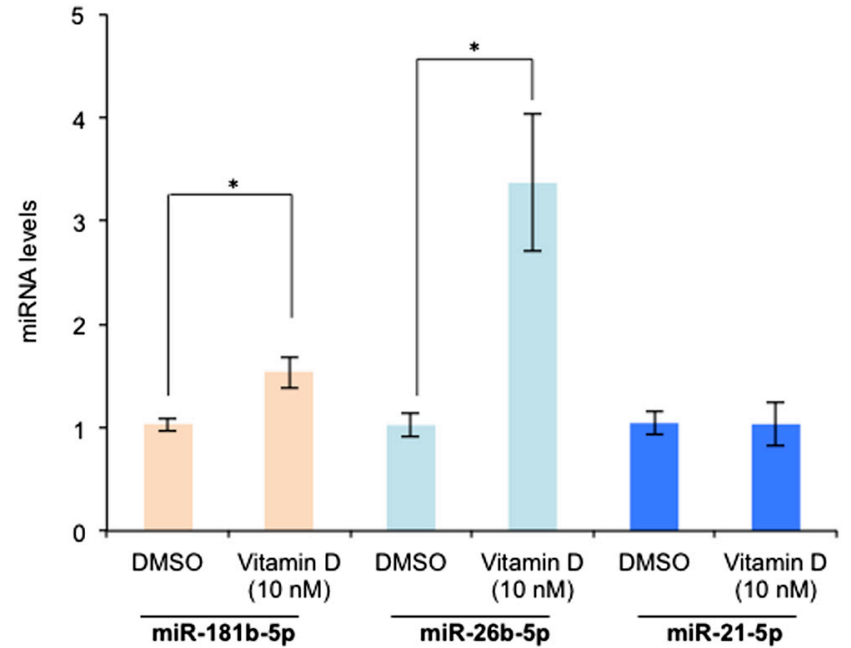

Figure 3

$1,25(\mathrm{OH}) 2 \mathrm{D}$ induces transcription of miR-181b-5p and miR-26b-5p in the human placenta. Term STB were exposed to $1,25(\mathrm{OH}) 2 \mathrm{D}$ at $10 \mathrm{nM}$ for $24 \mathrm{~h}$ and levels of miR-181b-5p, miR-26b-5p or the negative control miR-21-5p were assessed by RT-qPCR with normalization to the internal control U6 snRNA. Three independent experiments were performed. ${ }^{*} P<0.01$ (Student $t$ test, 2-tailed).

(Fig. 5A and B). We further mutated the miR-181b-5p(Fig. 4C) and miR-26b-5p-binding sites in the 3'-UTR of $C R H$ or COX-2 and found a loss of repression (Fig. 5C).

Taken together, the data suggest that $C R H$ is directly targeted by $m i R-181 b-5 p$ and COX-2 by $m i R-26 b-5 p$.

\section{Basal occupancy and 1,25(OH)2D-stimulated VDR binding in human STB}

In order to further confirm the role of $1,25(\mathrm{OH}) 2 \mathrm{D} / \mathrm{VDR}-$ miRNA signaling in regulation of pro-labor mediators in human STB, we compare peaks of MIR181B2 or MIR26B gene in human STB treated with DMSO or 1,25(OH)2D. Wiggle tracks were obtained from UCSC Browser (25). As shown in Fig. 6, peaks were called for either MIR181B2 or MIR26B following 1,25(OH)2D treatment.

\section{Discussion}

A better understanding of the molecular mechanisms underlying spontaneous preterm labor is essential for the development of better diagnostic tools and therapeutics to reduce the incidence of preterm deliveries and improve neonatal outcomes. There is strong evidence that CRH is a component of the clock that governs the length of pregnancy, and the role of COX-2 and prostaglandins in the initiation of labor has been extensively documented (26).

$\begin{array}{lr}\text { https://ec.bioscientifica.com } & \text { ○ } 2018 \text { The authors } \\ \text { https://doi.org/10.1530/EC-18-0345 } & \text { Published by Bioscientifica Ltd }\end{array}$

Here, we provide evidence that vitamin D-VDR signaling stimulates transcription of the miRNAs including miR-181b-2 and miR-26b, which cause post-translational inhibition of $C R H$ and $C O X-2$, respectively. These processes may support the observed association between vitamin D deficiency and preterm birth and reduction in preterm birth incidence with vitamin D supplementation. In our previous work, we have demonstrated that both $\mathrm{CRH}$ and $\mathrm{COX}-2$ are regulated by the non-canonical NF-KB pathway (19), but vitamin D appears to exert its effects independent of NF-KB here.

Vitamin D-stimulated VDR can inhibit LPS-induced activation of the canonical NF-KB signaling in a direct manner. However, the majority of preterm labor cases are not necessarily a consequence of bacterial infection. As such, we started by seeking to investigate the genomewide effects by vitamin D-VDR signaling in the human placenta with use of chromatin immunoprecipitation followed by DNA sequencing to study known mediators
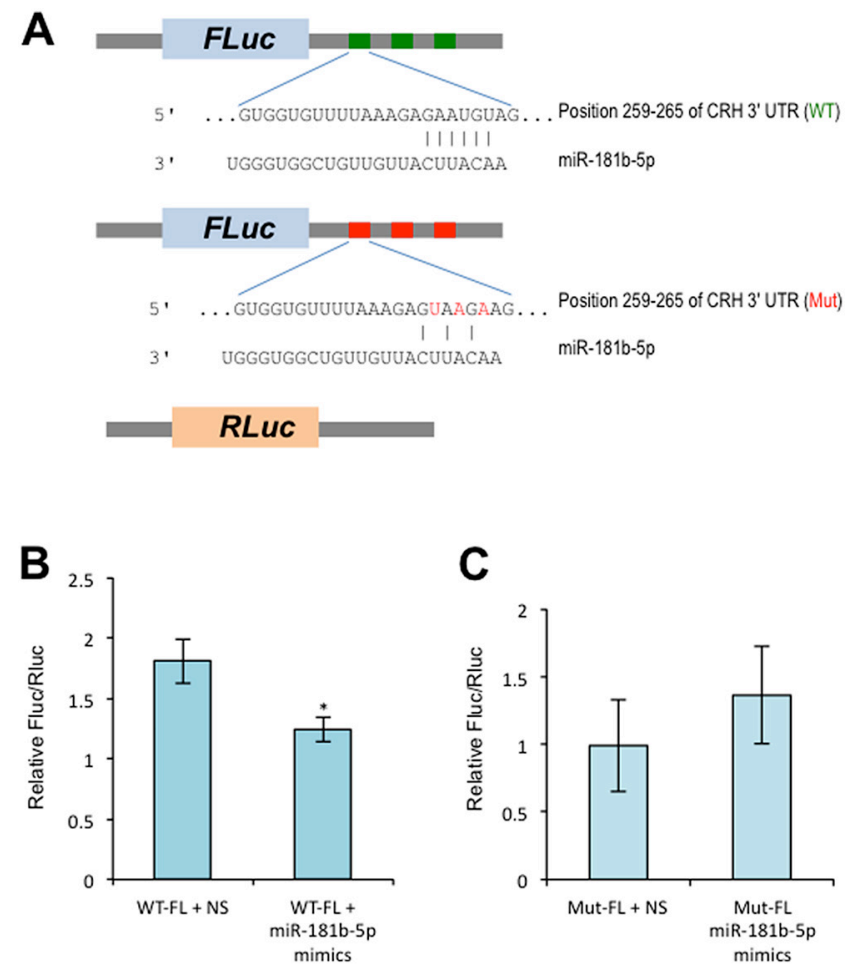

\section{Figure 4}

Validation of $\mathrm{CRH}$ targeted by miR-181b-5p with dual-luciferase assay. (A) Schematic presentation of DNA constructs as used in this assay. (B) Term STB were transiently transfected with WT constructs and miR-181b-5p mimics or non-specific miRNA mimics (NS) as indicated for $48 \mathrm{~h}$ followed by dual-luciferase assay. (C) Term STB was transiently transfected with mutant (Mut) constructs and miR-181b-5p mimics or non-specific miRNA mimics (NS) as indicated for $48 \mathrm{~h}$ followed by dual-luciferase assay. Three independent experiments were performed. $\star P<0.01$ (Student $t$ test, 2-tailed).

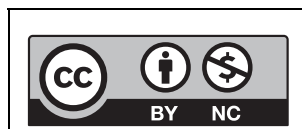

This work is licensed under a Creative Commons Attribution-NonCommercial 4.0 International License. 
A

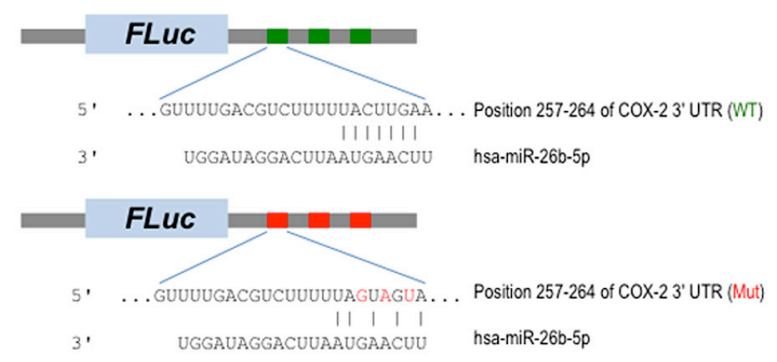

B

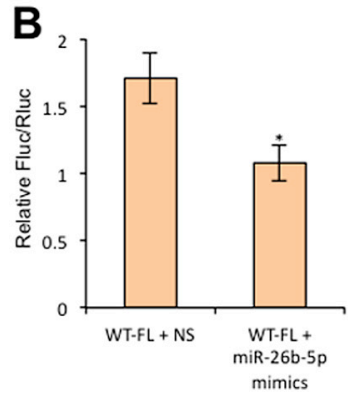

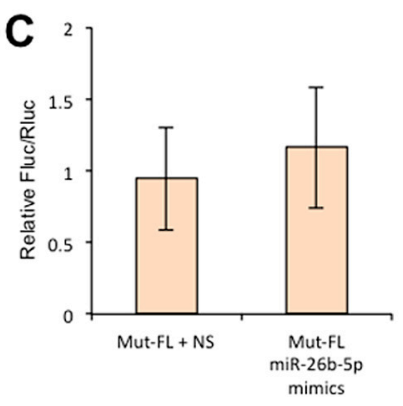

Figure 5

Validation of COX-2 targeted by miR-26b-5p with dual-luciferase assay. (A) Schematic presentation of DNA constructs as used in this assay. (B) Term STB were transiently transfected with WT constructs and miR-26b-5p mimics or non-specific miRNA mimics (NS) as indicated for $48 \mathrm{~h}$ followed by dual-luciferase assay. (C) Term STB was transiently transfected with mutant (Mut) constructs and miR-26b-5p mimics or non-specific miRNA mimics (NS) as indicated for $48 \mathrm{~h}$ followed by dual-luciferase assay. Three independent experiments were performed. $* P<0.01$ (Student $t$ test, 2-tailed).

of normal parturition. Such a genome-wide profiling of VDR-occupied genomic loci could further assist in clarification of mechanisms underlying their molecular action. In addition, our understanding of the biological functions of miRNA relies on the identification of relevant target genes. The majority of the miRNA-target sequence matching is based on imperfect complementarity of the
miRNA with the 3 '-UTRs of target mRNAs. As a result, combined computational prediction of miRNA targets and subsequent experimental validation would further justify our conclusions in this study.

Our data further suggest that apart from miR-181b-2 and miR-26, other miRNAs may also be involved in regulation of $\mathrm{CRH}, \mathrm{COX}-2$ and other labor-associated mediators. As described in Table 1, vitamin D-VDR also binds to genomic loci of multiple let-7s (-a, -e or -i) and those like miR-29a, miR-29c and miR-183. The predicted targets of let-7s include transcriptional factor STAT3, phospholipid platelet-activating factor (PAF) receptor (PTAFR) and NF-KB2 (p100/p52), and those of miR-29a, miR-29c and miR-183 include matrix metalloproteinase-2 (MMP-2), MMP-9 and oxytocin receptor (OXTR).

In the human placenta, cortisol promotes interaction of the glucocorticoid receptor with STAT3 to facilitate transcription of COX-2 (27), and we have shown that knockdown of STAT3 led to downregulation of both CRH and COX-2 in a dose-dependent manner (20). In addition, a recent study examining the role of fetal signals in initiation of parturition in the mouse demonstrates that surfactant protein-A (SP-A) and glycerophospholipid PAF secreted by mature fetal lungs led to a fall in progesterone that led to the onset of parturition in the mouse model (28), supporting the earlier observation that developing fetal lung-secreted PAF into amniotic fluid contributes to initiation of term or preterm labor (29). Moreover, extracellular matrix (ECM) remodeling has been implicated in many processes in human term and preterm labor, which include cervical ripening, fetal membrane rupture and placental detachment from the maternal uterus. Most of the ECM and basement membrane components can be degraded by matrix metalloproteinases, a group of structurally related zinc-dependent enzymes (30). These include MMP-2 and MMP-9, which are capable of

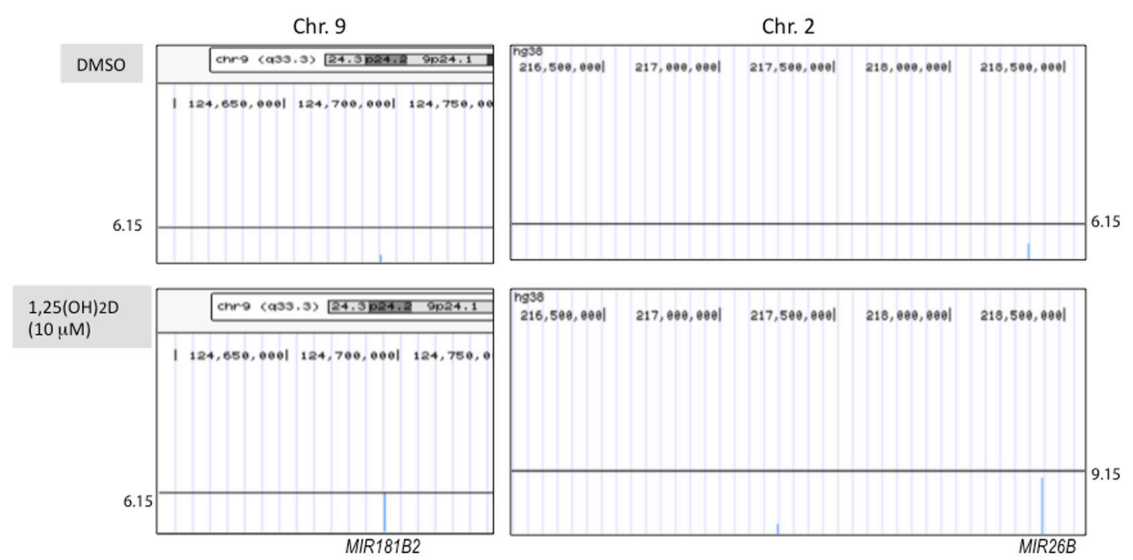

\section{Figure 6}

VDR ChIP-seq analysis for MIR181B2 and MIR26B. VDR ChIP-seq data shown for peaks of MIR181B2 (Chr9: 124693710...124693798) or MIR26B (Chr2: 218402637...218402746) in either resting (DMSO, $n=1)$ or after induction with 1,25(OH)2D for $24 \mathrm{~h}$ $(n=1)$, which were generated from UCSC Genome Browser tracks by uploaded wiggle files.

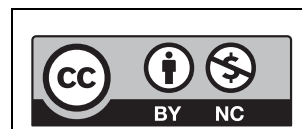

This work is licensed under a Creative Commons Attribution-NonCommercial 4.0 International License. 
digesting collagen IV, a major component of basement membrane. To this end, our future directions could include investigating the role of the miRNAs identified in this study in regulation of pro-labor mediators in the placenta as well as other gestational tissues.

In summary, this study has revealed a novel mechanism, by which vitamin $\mathrm{D}$ may contribute to reduced risk of preterm labor by regulation of $\mathrm{CRH}$ and other pro-labor mediators. These findings will provide significant insights into harnessing the medical benefits provided by $1,25(\mathrm{OH}) 2 \mathrm{D}-\mathrm{VDR}$ complex in future efforts.

\section{Supplementary data}

This is linked to the online version of the paper at https://doi.org/10.1530/ EC-18-0345.

\section{Declaration of interest}

The authors declare that there is no conflict of interest that could be perceived as prejudicing the impartiality of the research reported.

\section{Funding}

This study was in part (ChIP-seq and bioinformatics analysis) supported by an NIEHS grant (ES005022) to B W.

\section{References}

1 Bodnar LM, Klebanoff MA, Gernand AD, Platt RW, Parks WT, Catov JM \& Simhan HN. Maternal vitamin D status and spontaneous preterm birth by placental histology in the US Collaborative Perinatal Project. American Journal of Epidemiology 2014179 168-176. (https://doi.org/10.1093/aje/kwt237)

2 Bodnar LM, Platt RW \& Simhan HN. Early-pregnancy vitamin D deficiency and risk of preterm birth subtypes. Obstetrics and Gynecology 2015125 439-447. (https://doi.org/10.1097/ AOG.0000000000000621)

3 De-Regil LM, Palacios C, Lombardo LK \& Pena-Rosas JP. Vitamin D supplementation for women during pregnancy. Cochrane Database of Systematic Reviews 2016 CD008873. (https://doi. org/10.1002/14651858.CD008873.pub3)

4 Shin JS, Choi MY, Longtine MS \& Nelson DM. Vitamin D effects on pregnancy and the placenta. Placenta 201031 1027-1034. (https:// doi.org/10.1016/j.placenta.2010.08.015)

5 Shibasaki T, Odagiri E, Shizume K \& Ling N. Corticotropin-releasing factor-like activity in human placental extracts. Journal of Clinical Endocrinology and Metabolism 198255 384-386. (https://doi. org/10.1210/jcem-55-2-384)

6 McLean M, Bisits A, Davies J, Woods R, Lowry P \& Smith R. A placental clock controlling the length of human pregnancy. Nature Medicine 19951 460-463. (https://doi.org/10.1038/nm0595-460)

7 Wadhwa PD, Porto M, Garite TJ, Chicz-DeMet A \& Sandman CA. Maternal corticotropin-releasing hormone levels in the early third trimester predict length of gestation in human pregnancy. American Journal of Obstetrics and Gynecology 1998179 1079-1085. (https://doi. org/10.1016/S0002-9378(98)70219-4)

8 Hobel CJ, Dunkel-Schetter C, Roesch SC, Castro LC \& Arora CP. Maternal plasma corticotropin-releasing hormone associated with stress at 20 weeks' gestation in pregnancies ending in preterm delivery. American Journal of Obstetrics and Gynecology 1999180 S257-S263. (https://doi.org/10.1016/S0002-9378(99)70712-X)

9 Warren WB, Patrick SL \& Goland RS. Elevated maternal plasma corticotropin-releasing hormone levels in pregnancies complicated by preterm labor. American Journal of Obstetrics and Gynecology 1992 166 1198-1204; discussion 1204-1197. (https://doi.org/10.1016/ S0002-9378(11)90606-1)

10 Mohamed SA, El Andaloussi A, Al-Hendy A, Menon R, Behnia F, Schulkin J \& Power ML. Vitamin D and corticotropin-releasing hormone in term and preterm birth: potential contributions to preterm labor and birth outcome. Journal of Maternal-Fetal and Neonatal Medicine 201731 2911-2917. (https://doi.org/10.1080/1476 7058.2017.1359534)

11 Schulkin J. The CRF Signal: Uncovering an Information Molecule, 1st ed. Oxford, New York, NY, USA: Oxford University Press, 2017.

12 Karim SM \& Hillier K. Prostaglandins in the control of animal and human reproduction. British Medical Bulletin 197935 173-180. (https://doi.org/10.1093/oxfordjournals.bmb.a071566)

13 Di Stefano V, Wang B, Parobchak N, Roche N \& Rosen T. RelB/p52mediated NF-kappaB signaling alters histone acetylation to increase the abundance of corticotropin-releasing hormone in human placenta. Science Signaling 20158 ra85. (https://doi.org/10.1126/ scisignal.aaa9806)

14 Mizwicki MT \& Norman AW. The vitamin D sterol-vitamin D receptor ensemble model offers unique insights into both genomic and rapid-response signaling. Science Signaling 20092 re4. (https:// doi.org/10.1126/scisignal.275re4)

15 Chen Y, Zhang J, Ge X, Du J, Deb DK \& Li YC. Vitamin D receptor inhibits nuclear factor kappaB activation by interacting with IkappaB kinase beta protein. Journal of Biological Chemistry 2013288 19450-19458. (https://doi.org/10.1074/jbc. M113.467670)

16 Robertson SA, Skinner RJ \& Care AS. Essential role for IL-10 in resistance to lipopolysaccharide-induced preterm labor in mice. Journal of Immunology 2006177 4888-4896. (https://doi.org/10.4049/ jimmunol.177.7.4888)

17 Thota C, Farmer T, Garfield RE, Menon R \& Al-Hendy A. Vitamin D elicits anti-inflammatory response, inhibits contractileassociated proteins, and modulates Toll-like receptors in human myometrial cells. Reproductive Sciences 201320 463-475. (https://doi. org/10.1177/1933719112459225)

18 Uhlen M, Fagerberg L, Hallstrom BM, Lindskog C, Oksvold P, Mardinoglu A, Sivertsson A, Kampf C, Sjostedt E, Asplund A, et al. Proteomics. Tissue-based map of the human proteome. Science 2015 347 1260419. (https://doi.org/10.1126/science.1260419)

19 Wang B, Parobchak N \& Rosen T. RelB/NF-kappaB2 regulates corticotropin-releasing hormone in the human placenta. Molecular Endocrinology 201226 1356-1369. (https://doi.org/10.1210/me.20121035)

20 Yu LJ, Wang B, Parobchak N, Roche N \& Rosen T. STAT3 cooperates with the non-canonical NF-kappaB signaling to regulate pro-labor genes in the human placenta. Placenta 201536 581-586. (https://doi. org/10.1016/j.placenta.2015.02.013)

21 Wienholds E \& Plasterk RH. MicroRNA function in animal development. FEBS Letters 2005579 5911-5922. (https://doi. org/10.1016/j.febslet.2005.07.070)

22 Piriyapongsa J, Jordan IK, Conley AB, Ronan T \& Smalheiser NR. Transcription factor binding sites are highly enriched within microRNA precursor sequences. Biology Direct 20116 61. (https://doi. org/10.1186/1745-6150-6-61)

23 Agarwal V, Bell GW, Nam JW \& Bartel DP. Predicting effective microRNA target sites in mammalian mRNAs. Elife 20154 e05005. (https://doi.org/10.7554/eLife.05005)

24 Kozomara A \& Griffiths-Jones S. miRBase: annotating high confidence microRNAs using deep sequencing data. Nucleic Acids Research 201442 D68-D73. (https://doi.org/10.1093/nar/gkt1181) 
25 Casper J, Zweig AS, Villarreal C, Tyner C, Speir ML, Rosenbloom KR, Raney BJ, Lee CM, Lee BT, Karolchik D, et al. The UCSC Genome Browser database: 2018 update. Nucleic Acids Research 201846 D762-D769. (https://doi.org/10.1093/nar/gkx1020)

26 Grigsby PL, Sooranna SR, Brockman DE, Johnson MR \& Myatt L. Localization and expression of prostaglandin $\mathrm{E} 2$ receptors in human placenta and corresponding fetal membranes with labor. American Journal of Obstetrics and Gynecology 2006195 260-269. (https://doi. org/10.1016/j.ajog.2006.01.082)

27 Wang W, Guo C, Zhu P, Lu J, Li W, Liu C, Xie H, Myatt L, Chen ZJ $\&$ Sun K. Phosphorylation of STAT3 mediates the induction of cyclooxygenase- 2 by cortisol in the human amnion at parturition Science Signaling 20158 ra106. (https://doi.org/10.1126/scisignal. aac6151)
28 Gao L, Rabbitt EH, Condon JC, Renthal NE, Johnston JM, Mitsche MA, Chambon P, Xu J, O'Malley BW \& Mendelson CR. Steroid receptor coactivators 1 and 2 mediate fetal-to-maternal signaling that initiates parturition. Journal of Clinical Investigation 2015125 2808-2824. (https://doi.org/ 10.1172/JCI78544)

29 Yasuda K, Furukawa M \& Johnston JM. Effect of estrogens on plasma platelet-activating factor acetylhydrolase and the timing of parturition in the rat. Biology of Reproduction $1996 \mathbf{5 4} 224-229$. (https://doi.org/10.1095/biolreprod54.1.224)

30 Lu P, Takai K, Weaver VM \& Werb Z. Extracellular matrix degradation and remodeling in development and disease. Cold Spring Harbor Perspectives in Biology 20113 a005058. (https://doi.org/10.1101/ cshperspect.a005058)

\section{Received in final form 23 October 2018}

Accepted 5 November 2018

Accepted Preprint published online 5 November 2018 https://ec.bioscientifica.com https://doi.org/10.1530/EC-18-0345 (c) 2018 The authors Published by Bioscientifica Ltd

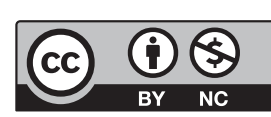

This work is licensed under a Creative Commons Attribution-NonCommercial 4.0 International License. 